\title{
What Is the Universe Ultimately Made of?
}

\author{
Matsuo Sekine* \\ Tokyo, Japan \\ Email: fwnn4573@nifty.com
}

How to cite this paper: Sekine, M. (2021) What Is the Universe Ultimately Made of? Journal of High Energy Physics, Gravitation and Cosmology, 7, 1161-1181. https://doi.org/10.4236/jhepgc.2021.73068

Received: June 8, 2021

Accepted: July 17, 2021

Published: July 20, 2021

Copyright () 2021 by author(s) and Scientific Research Publishing Inc. This work is licensed under the Creative Commons Attribution International License (CC BY 4.0).

http://creativecommons.org/licenses/by/4.0/

\section{(c) (i) Open Access}

\begin{abstract}
As the ultimate building blocks of the universe, the limit structureless quark $u_{\infty}$ and its anti-quark $d_{\infty}=\overline{u_{\infty}}=u_{\infty}^{C P}$ are considered at the infinite sublayer level of the quark model. Then $C P$ is violated in the doublet of $u_{\infty}$ and $u_{\infty}^{C P}$ quarks to account for the asymmetry of the number of particles and antiparticles. This $C P$ violation is explained by a $S U(2)$ noncommutative geometry. The second, third and fourth generation quarks are considered only as the excited states of the first generation $u_{\infty}$ and $u_{\infty}^{C P}$ quarks. The fourth generation quarks are derived from both $C P T$ transformation and the $S U(2)_{L} \times U(1)$ gauge theory. The dark matter, quarks, leptons, gauge bosons and Higgs bosons are composed of only the $u_{\infty}$ and $u_{\infty}^{C P}$ quarks and the cosmological constant in Einstein's field equation is also derived from the Higgs potential. Thus, the limit particle $u_{\infty}$ and its anti-particle $u_{\infty}^{C P}$ are the ultimate particles of the universe and produced thermally in the hot early universe of the Big Bang.
\end{abstract}

\section{Keywords}

Non-Baryonic Quark, Dark Matter, Gauge Bosons, Higgs Bosons

Cosmological Constant

\section{Introduction}

The present author has proposed an infinite sub-layer quark model [1] and showed that there exists an infinite number of non-baryonic and half-electric charged $u_{\infty}$ and $u_{\infty}^{c p}$ quarks at an infinite sub-layer level. The superscript $C P$ means charge conjugation and parity transformation. The ultimate particle $u_{\infty}$ has all one-half quantum numbers of spin $S=1 / 2$, isospin $I=1 / 2$, the third component of isospin $I_{3}=-1 / 2$ and fractional electric charge $Q=(1 / 2)|e|$

${ }^{\star}$ Note: Matsuo Sekine was with the Tokyo Institute of Technology and the National Defense Academy of Japan. 
where $e$ is the electron charge. The infinite sub-layer quark model was based on fractal self-similarities and implied that the proton $(p)$ and the neutron $(n)$ are made up of $u_{1}$ and $d_{1}$ quarks, so that $p=u_{1} u_{1} d_{1}$ and $u=u_{1} d_{1} d_{1}$. Furthermore, $u_{1}$ and $d_{1}$ quarks are made up of $u_{2}$ and $d_{2}$, etc. This is illustrated in Figure 1.

In summary, $u_{N}$ and $d_{N}$ quarks at level $N$ are made up of $u_{N+1}$ and $d_{N+1}$ quarks at level $N+1$, such as $u_{N}=\left(u_{N+1}, u_{N+1}, d_{N+1}\right)$ and $d_{N}=\left(u_{N+1}, d_{N+1}, d_{N+1}\right)$ where

$$
N=1,2,3, \cdots, \infty .
$$

Here, the $u_{N}$ and $d_{N}$ quarks have quantum numbers of spin $S=1 / 2$, isospin $I=1 / 2$, baryon number $B=1 / 3^{N}$, third component of isospin $I_{3}=+1 / 2$ and fractional electric charge $Q=\frac{1+3^{N}}{2 \times 3^{N}}|e|$ for he $u_{N}$ quark, and $I_{3}=-1 / 2$ and $Q=\frac{1-3^{N}}{2 \times 3^{N}}|e|$ for the $d_{N}$ quark. The fractional electric charge is derived from the Gell-Mann-Nishijima formula, $Q=I_{3}+B / 2$ [2]. The antiparticle of $u_{\infty}$ is the $d_{\infty}$ quark, since the baryon number vanishes at $N=\infty$. The number of quarks at level $N$ is $3^{N}$. Thus, at $N=\infty$, an infinite number of point-like quarks $\left(u_{\infty}\right)$ and anti-quarks $\left(d_{\infty}=\overline{u_{\infty}}=u_{\infty}^{C P}\right)$ is considered as constituting the nucleon. The superscript $C P$ means charge conjugation and parity transformation. The ultimate particle $u_{\infty}$ has quantum numbers of $S=1 / 2$, $I=1 / 2, \quad I_{3}=1 / 2$ and $Q=(1 / 2)|e|$. Thus, all quantum numbers of the $u_{\infty}$ quark are just one-half and this quark is non-baryonic, since the baryon number $B$ is zero at an infinite sub-layer level. In a previous paper [3], we proposed the non-baryonic and exotic quark $u_{\infty}$ as an excellent candidate for non-baryonic cold dark matter to comprise the universe, since they are absolutely stable and the non-baryonic particles with the baryon number 0 . In this model, a pair of an infinite number of $u_{\infty}$ and $u_{\infty}^{C P}$ quarks can be produced thermally in the hot early universe of the Big Bang and form the nucleons, and leave approximately the right relic abundance to account for the observed dark matter. Also, in this paper, we will show that $C P$ is violated in only doublet of the ultimate quarks $u_{\infty}$ and anti-quarks $u_{\infty}^{C P}$ to account for the asymmetry of particles and anti-particles in the present universe.

To this end, we considered the $S U(2)$ noncommutative geometry from our published paper [4] and our published book [5].

Now we see the higher hierarchy of clusters from quarks to galaxies. This is also based on fractal and self-similarities similar to the infinite sub-layer quark model. For example, as we move to the lower side of the hierarchy of structures, we studied an infinite sublayer quark model. On the other hand, attempts have been made to model the higher side of the hierarchy of structures of the universe through fractal-like geometry. This is shown in Figure 2.

$N_{1}$ stars form a galaxy $G_{1}$ with radius $R_{1}, N_{2}$ galaxies form a second order galaxy $G_{2}$ with radius $R_{2}, \cdots, N_{i}$ galaxies form the $i$-th order galaxy 


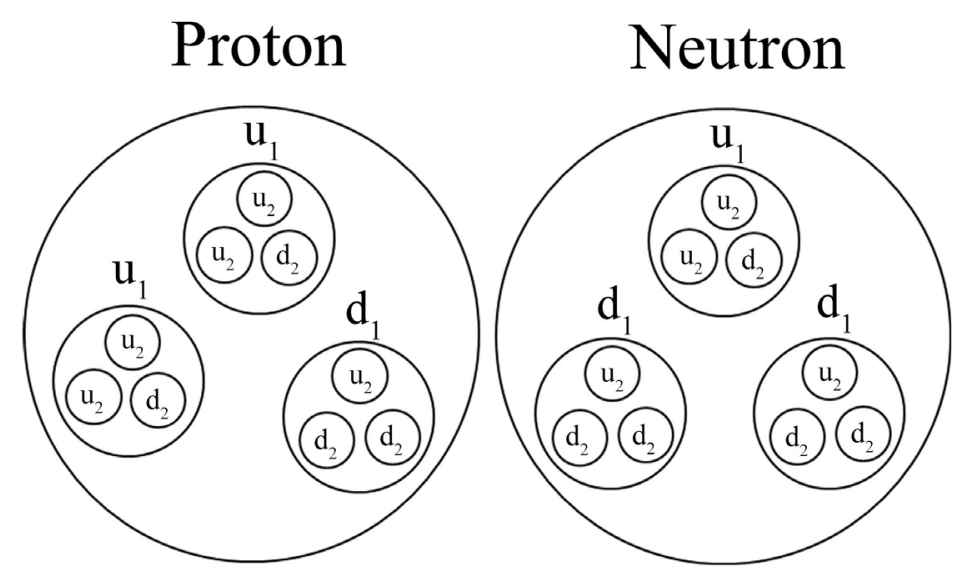

Figure 1. Proton is made up of $u_{1} u_{1} d_{1}$ and neutron $u_{1} d_{1} d_{1}$. Furthermore, $u_{1}$ quarkis made up of $u_{2} u_{2} d_{2}$ and $d_{1}$ quark $u_{2} d_{2} d_{2}$.

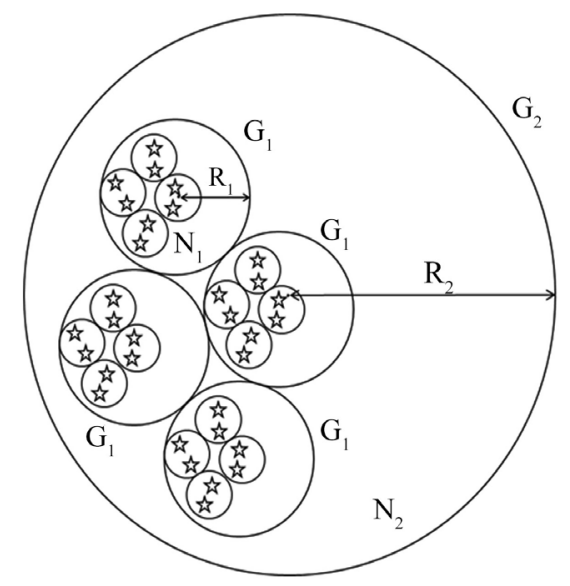

Figure 2. Fractal-like universe.

$G_{i}$ with radius $R_{i}, \cdots$, and infinitum. The Swedish astronomer Charlier showed that if the condition $R_{i} / R_{i-1}>\sqrt{N_{i}}$ is satisfied, we can avoid the following Olbers paradox [6]: In 1826, Olbers had remarked that, if the universe were infinite and filled uniformly with stars, then the sky should glow as brightly as the face of the sun [7]. In modern cosmology, this paradox is explained by the expansion of the universe.

According to Alfvén, the hierarchical structure theory of the universe does not necessarily come into conflict with the Big Bang theory [8]. Thus, the infinite sub-layer quark model is similar to the hierarchical fractal-like universe. In a previous paper [9], the present author proposed the Cantor set quark model as an alternative to the infinite sub-layer quark model. Then we introduce the colors. we introduce the color charges of $\operatorname{red}(\mathrm{R})$, green $(\mathrm{G})$ and blue(B) in quantum chromodynamics (QCD) and propose that the proton and the neutron are made of an infinite number of $u_{\infty}^{R} u_{\infty}^{G} d_{\infty}^{B}, u_{\infty}^{R} u_{\infty}^{B} d_{\infty}^{G}, u_{\infty}^{B} u_{\infty}^{G} d_{\infty}^{R}$ and $d_{\infty}^{R} d_{\infty}^{G} u_{\infty}^{B}, d_{\infty}^{R} d_{\infty}^{B} u_{\infty}^{G}$, $d_{\infty}^{B} d_{\infty}^{G} u_{\infty}^{R}$. The color-neutral system, that is, "white" color requires $\mathrm{R}+\mathrm{G}+\mathrm{B}=1$ with $1 / 3$ color charge quantum number for each $R, G, B$ color charges.

In the following, we shall apply the Cantor set to the quark model without in- 
troducing the color charges. In both the Cantor model and an infinite sublayer model, there exists an infinite number of $u_{\infty}$ and $u_{\infty}^{c p}$ quarks.

Then we compare the electron-positron measurements with the prediction from $u_{\infty}$ and $u_{\infty}^{c p}$ quarks. Finally, it is shown that the dark matter, leptons, gauge bosons and Higgs bosons are composed of $u_{\infty}$ and $u_{\infty}^{c p}$ quarks.

Thus, the Higgs bosons are composed of $u_{\infty}$ and $u_{\infty}^{C P}$ dark matter particles and give the masses to gauge bosons, quarks and leptons in the framework of the standard $S U(2)_{L} \times U(1)$ electroweak model. Moreover, we will replace the Higgs potential by the gravitational potential and it is then shown that the masses are produced and a cosmological constant is derived [10]. It was then emphasized that if we can write down the $n$th order T product Green's function in the path-integral representation, then it is expected to construct a quantization theory including the cosmological constant. This prescription of the quantization by path-integral representation without the gravitational field is suggested from our published paper [11] and our published book [12].

\section{The Cantor Set Constructed from an Infinite Number of Quarks}

We will show that the Cantor set [13] is constructed from an infinite number of point-like $u_{\infty}$ and $u_{\infty}^{C P}$ quarks. The Cantor middle-thirds or ternary set is described by repeatedly removing the open middle thirds of a set of line segments. Begin with the interval $[0,1]$ and divide it into three equal open intervals, that is, $\left[0, \frac{1}{3}\right],\left[\frac{1}{3}, \frac{2}{3}\right],\left[\frac{2}{3}, 1\right]$. Remove the open middle third $\left(\frac{1}{3}, \frac{2}{3}\right)$, leaving two line segments: $\left[0, \frac{1}{3}\right] \cup\left[\frac{2}{3}, 1\right]$. Next, subdivide each of these two remaining intervals into three equal open subintervals and again delete the open middle third of each of these remaining intervals, and so on, ad infinitum. In this infinite process, finally, the Cantor set contains an infinite number of points. The Cantor set is an example of fractal and self-similarity [4]. We apply this process to the infinite sub-layer quark model. We begin with $\left(u_{1}, u_{1}{ }^{M}, d_{1}\right)$ quarks which constitute the proton. Here the superscript $M$ means the middle quark between $u_{1}$ and $d_{1}$ quarks. Now we remove the $u_{1}{ }^{M}$ quark, leaving $\left(u_{1}, d_{1}\right)$ behind. Next, the $u_{1}$ and $d_{1}$ quarks are subdivided into $u_{1}=\left(u_{2}, u_{2}{ }^{M}, d_{2}\right)$ and $d_{1}=\left(u_{2}, d_{2}{ }^{M}, d_{2}\right)$ and again we remove the $u_{2}{ }^{M}$ and $d_{2}{ }^{M}$ quarks, leaving us with four quarks $\left(\left(u_{2}, d_{2}\right),\left(u_{2}, d_{2}\right)\right)$. This process is to be continued infinitely. The number of leaving quarks at level $N$ is $2^{N}$ where $N=1,2,3, \cdots, \infty$. Thus, at $N=\infty$, there exists an infinite number of point-like quarks $\left(u_{\infty}\right)$ and anti-quarks $\left(u_{\infty}^{C P}\right)$. Finally, it is concluded that an infinite number of point-like $u_{\infty}$ and $u_{\infty}^{C P}$ quarks that remain after all these middle quarks have been removed is called the Cantor set. The removed middle quarks are arranged as follows:

$$
u_{1}{ }^{M},\left(u_{2}{ }^{M}+d_{2}{ }^{M}\right), 2\left(u_{3}{ }^{M}+d_{3}{ }^{M}\right), 2^{2}\left(u_{4}{ }^{M}+d_{4}{ }^{M}\right), \cdots, 2^{N-2}\left(u_{N}{ }^{M}+d_{N}{ }^{M}\right), \cdots
$$

where $N=2,3, \cdots, \infty$. At $N=\infty$, there exists an infinite number of point-like 
quarks $\left(u_{\infty}\right)^{M}$ and anti-quarks $\left(u_{\infty}^{C P}\right)^{M}$ with all half-quantum numbers. We will show that the proton is constructed from these removed quarks of (1).

First, consider the baryon number. If we add up the baryon number from (1), we obtain $\frac{1}{3}+\frac{2}{9}+\frac{4}{27}+\cdots=1$. This total is the geometric progression. The electric charge $Q$ is calculated from (1) as

$$
Q=\frac{2}{3}|e|+\left(\frac{1}{9}|e|+\frac{2}{27}|e|+\frac{4}{81}|e|+\cdots\right)=\frac{2}{3}|e|+\frac{1}{3}|e|=|e| .
$$

Thus, the constituents from the removed middle quarks of (1) are the proton. Namely, the proton $p=\left(u_{1}, u_{1}{ }^{M}, d_{1}\right)$ is corresponding to $p=\left(u_{1}{ }^{M},\left(u_{2}{ }^{M}+d_{2}{ }^{M}\right)\right.$, $2\left(u_{3}{ }^{M}+d_{3}{ }^{M}\right), 2^{2}\left(u_{4}{ }^{M}+d_{4}{ }^{M}\right), \cdots, 2^{N-2}\left(u_{N}{ }^{M}+d_{N}{ }^{M}\right), \cdots$, an infinite number of point-like quarks $\left(u_{\infty}\right)^{M}$ and anti-quarks $\left.\left(u_{\infty}^{C P}\right)^{M}\right)$.

Now consider $\left(u_{1}, d_{1}^{M}, d_{1}\right)$ quarks which constitute the neutron. In the same way to the $\left(u_{1}, u_{1}{ }^{M}, d_{1}\right)$ quarks, we repeat any step in this infinite process. The removed quarks are as follows:

$$
d_{1}{ }^{M},\left(u_{2}{ }^{M}+d_{2}{ }^{M}\right), 2\left(u_{3}{ }^{M}+d_{3}{ }^{M}\right), 2^{2}\left(u_{4}{ }^{M}+d_{4}{ }^{M}\right), \cdots, 2^{N-2}\left(u_{N}{ }^{M}+d_{N}{ }^{M}\right), \cdots
$$

where $N=2,3, \cdots, \infty$. At $N=\infty$, there exists also an infinite number of pointlike quarks $\left(u_{\infty}\right)^{M}$ and anti-quarks $\left(u_{\infty}^{C P}\right)^{M}$ having all half-quantum numbers.

From (2), the baryon number is calculated as $\frac{1}{3}+\frac{2}{9}+\frac{4}{27}+\cdots=1$ and the electric charge $Q$ is $Q=-\frac{1}{3}|e|+\left(\frac{1}{9}|e|+\frac{2}{27}|e|+\frac{4}{81}|e|+\cdots\right)=-\frac{1}{3}|e|+\frac{1}{3}|e|=0$.

Thus, the constituents from the middle removed quarks of (2) are the neutron. Namely, the neutron $n=\left(u_{1}, d_{1}{ }^{M}, d_{1}\right)$ is corresponding to $n=\left(d_{1}{ }^{M},\left(u_{2}{ }^{M}+d_{2}{ }^{M}\right)\right.$, $2\left(u_{3}{ }^{M}+d_{3}{ }^{M}\right), 2^{2}\left(u_{4}{ }^{M}+d_{4}{ }^{M}\right), \cdots, 2^{N-2}\left(u_{N}{ }^{M}+d_{N}{ }^{M}\right), \cdots$, an infinite number of point-like quarks $\left(u_{\infty}\right)^{M}$ and anti-quarks $\left.\left(u_{\infty}^{C P}\right)^{M}\right)$.

As shown in Figure 3, this is pictured as a solar or atom system, with the sun corresponding to the nucleus, and the orbiting planets to the electrons. It is amazing to note that there exists an infinite number of the ultimate quark $u_{\infty}$ and anti-quark $d_{\infty}=\bar{u}_{\infty}=u_{\infty}^{C P}$ in the orbitor shell of the infinity. Thus, a miniature solar system is so ubiquitous in nature. Recently, there has been great interest in the atomic shell model in a field of quantum mechanics, elementary particle physics and quantum gravity. For example, Bohr's classical atomic model was applied to Black holes [14]. It was then shown that the radiation transition from one allowed shell to another shell is related to the Hawking radiation.

\section{Experimental Evidence for the Ultimate $u_{\infty}$ and $\boldsymbol{u}_{\infty}^{C P}$ Quarks}

We will examine the cross-section ratio $R$ in electron-positron annihilation into muon pairs and quark pairs.

This is shown in Figure 4. 


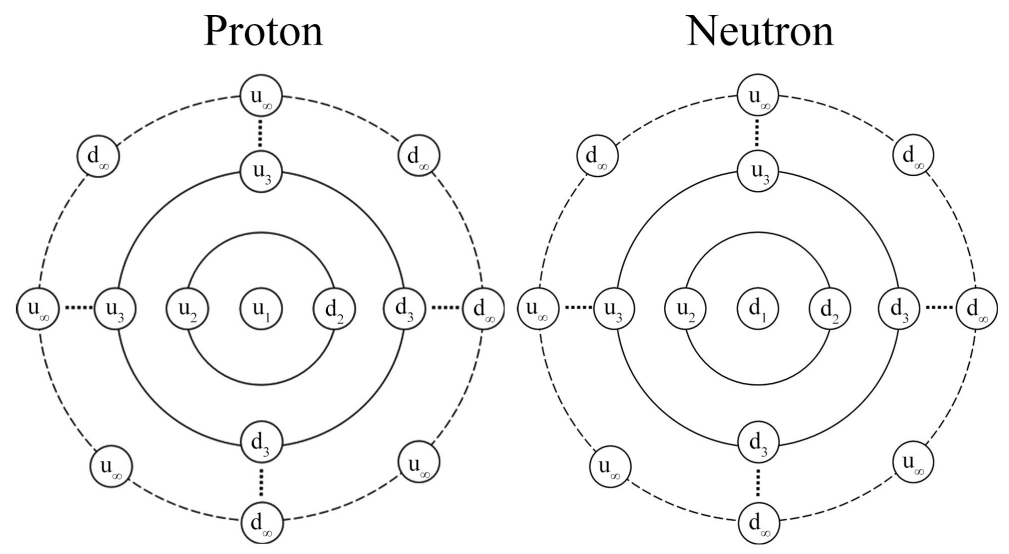

Figure 3. Atomic shell model of the structure of the proton and the neutron derived from the Cantor set.

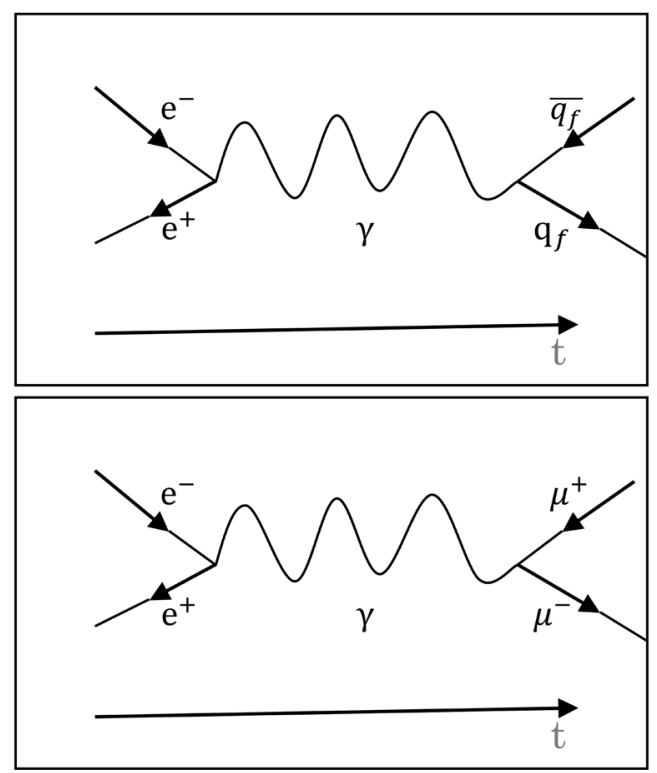

Figure 4. Electron-positron annihilation into muon pairs and quark pairs.

The lowest order QED total cross-section for the process via a virtual photon $(\gamma) e^{+} e^{-} \rightarrow \gamma \rightarrow \mu^{+} \mu^{-}$gives

$$
\sigma=\frac{4 \pi \alpha^{2}}{3(\sqrt{s})^{2}}=\frac{86.8}{(\sqrt{s})^{2}}(\mathrm{nb})
$$

where $\alpha$ is the fine structure constant and $\sqrt{s}$ is the center-of-mass energy [2].

We neglected the lepton masses. An $e^{+} e^{-}$annihilation can produce hadrons through a virtual photon $(\gamma)$ and $e^{+} e^{-} \rightarrow \gamma \rightarrow q_{f}(\infty) \overline{q_{f}}(\infty) \rightarrow$ hadrons .

We obtain the total cross section

$$
\sigma=\frac{4 \pi \alpha^{2}}{3(\sqrt{s})^{2}} Q_{f}^{2} N_{c}
$$


Here $Q_{f}$ are quark charges for the flavors $f=u, d, s, c, b$ and $t . N_{c}$ are the color charges $c=$ red, green and blue and $N_{c}=3$.

The cross section ratio $R$ for quarks $q_{f}(\infty)$ is written as

$$
R=\frac{\sigma\left(e^{+} e^{-} \rightarrow q_{f}(\infty) \bar{q}_{f}(\infty)\right)}{\sigma\left(e^{+} e^{-} \rightarrow \mu^{+} \mu^{-}\right)}=3 \sum_{q_{f}(\infty)} Q_{q_{f}(\infty)}^{2}
$$

From Table 1 and the following generalized Gell-Mann-Nishizima formula,

$$
Q=I_{3}+\frac{1}{2}(B+S+C+\mathcal{B}+T) \text {. }
$$

We obtain the following for cross section ratio $R$ for $u, d, s, c$ and $b$ quarks:

$$
\begin{aligned}
R=3 \times & {\left[Q_{u}^{2}+Q_{d}^{2}+Q_{s}^{2}\right]=3 \times\left[\left(\frac{2}{3}\right)^{2}+\left(-\frac{1}{3}\right)^{2}+\left(-\frac{1}{3}\right)^{2}\right]=\frac{6}{3}=2 \text { for } u, d, s \text { quarks } } \\
R & =3 \times\left[Q_{u}^{2}+Q_{d}^{2}+Q_{s}^{2}+Q_{c}^{2}\right] \\
& =3 \times\left[\left(\frac{2}{3}\right)^{2}+\left(-\frac{1}{3}\right)^{2}+\left(-\frac{1}{3}\right)^{2}+\left(\frac{2}{3}\right)^{2}\right]=\frac{10}{3} \text { for } u, d, s, c \text { quarks (6) } \\
R=3 \times\left[Q_{u}^{2}+Q_{d}^{2}+Q_{s}^{2}+Q_{c}^{2}+Q_{b}^{2}\right] & {\left[\left(\frac{2}{3}\right)^{2}+\left(-\frac{1}{3}\right)^{2}+\left(-\frac{1}{3}\right)^{2}+\left(\frac{2}{3}\right)^{2}+\left(-\frac{1}{3}\right)^{2}\right]=\frac{11}{3} \text { for } u, d, s, c, b \text { quarks } }
\end{aligned}
$$

From $u, d, s, c$ and $b$ quarks, we obtain the theoretical branching ratio $R=$ $11 / 3=3.67$. The third order QCD radiation correction formula is written as

$$
R=3 \sum_{q_{f}} Q_{q_{f}}^{2}\left[1+\left(\frac{\alpha_{s}(s)}{\pi}\right)+1.4092\left(\frac{\alpha_{s}(s)}{\pi}\right)^{2}-12.8046\left(\frac{\alpha_{s}(s)}{\pi}\right)^{3}\right]
$$

and gives $R(34 \mathrm{GeV})=11 / 3(1.056 \pm 0.008)=3.87 \pm 0.03$, thus the $\mathrm{QCD}$ correction increases the predicted value by $\sim 5 \%$ [15] which agrees with our predicated value $R=15 / 4=3.75$ from an infinite number of quarks and anti-quarks model better than the naïve 5 quark value $R=11 / 3=3.67$.

Thus, from Table 2, we obtain

$$
\begin{aligned}
& R=3 \times\left[\left(Q_{u}(\infty)\right)^{2}+\left(Q_{d}(\infty)\right)^{2}+\left(Q_{s}(\infty)\right)^{2}\right] \\
&=3 \times\left[\left(\frac{1}{2}\right)^{2}+\left(-\frac{1}{2}\right)^{2}+\left(-\frac{1}{2}\right)^{2}\right]=\frac{9}{4} \text { for } u(\infty), d(\infty), s(\infty) \text { quarks } \\
& R=3 \times\left[\left(Q_{u}(\infty)\right)^{2}+\left(Q_{d}(\infty)\right)^{2}+\left(Q_{s}(\infty)\right)^{2}+\left(Q_{c}(\infty)\right)^{2}\right] \\
&=3 \times\left[\left(\frac{1}{2}\right)^{2}+\left(-\frac{1}{2}\right)^{2}+\left(-\frac{1}{2}\right)^{2}+\left(\frac{1}{2}\right)^{2}\right]=\frac{12}{4}=3 \\
& R=3 \times\left[\left(Q_{u}(\infty)\right)^{2}+\left(Q_{d}(\infty)\right)^{2}+\left(Q_{s}(\infty)\right)^{2}+\left(Q_{c}(\infty)\right)^{2}+\left(Q_{b}(\infty)\right)^{2}\right] \\
&=3 \times\left[\left(\frac{1}{2}\right)^{2}+\left(-\frac{1}{2}\right)^{2}+\left(-\frac{1}{2}\right)^{2}+\left(\frac{1}{2}\right)^{2}+\left(-\frac{1}{2}\right)^{2}\right]=\frac{15}{4}=3.75 \\
& \text { for } u(\infty), d(\infty), s(\infty), c(\infty), b(\infty) \text { quarks. }
\end{aligned}
$$


Table 1. Additive quantum numbers of the quarks in the standard model. The subscript "L" indicates the left-handed particle.

\begin{tabular}{|c|c|c|c|c|c|c|}
\hline \multirow[b]{2}{*}{ Flavors } & \multicolumn{2}{|c|}{ First generation } & \multicolumn{2}{|c|}{ Secondgeneration } & \multicolumn{2}{|c|}{ Third generation } \\
\hline & $d$ & $u$ & $s$ & $c$ & $b$ & $t$ \\
\hline Baryon number $B$ & $1 / 3$ & $1 / 3$ & $1 / 3$ & $1 / 3$ & $1 / 3$ & $1 / 3$ \\
\hline Electric charge $Q$ & $-1 / 3$ & $2 / 3$ & $-1 / 3$ & $2 / 3$ & $-1 / 3$ & $2 / 3$ \\
\hline Isospin $I$ & $1 / 2$ & $1 / 2$ & 0 & 0 & 0 & 0 \\
\hline Third component of isospin $I_{3}$ & $-1 / 2$ & $1 / 2$ & 0 & 0 & 0 & 0 \\
\hline Strangeness number $S$ & 0 & 0 & -1 & 0 & 0 & 0 \\
\hline Charm number $C$ & 0 & 0 & 0 & 1 & 0 & 0 \\
\hline Bottomness number $\mathcal{B}$ & 0 & 0 & 0 & 0 & -1 & 0 \\
\hline Topness number $T$ & 0 & 0 & 0 & 0 & 0 & 1 \\
\hline Third component of weak isospin $\left(t_{3}\right)_{L}$ & $-1 / 2$ & $1 / 2$ & $-1 / 2$ & $1 / 2$ & $-1 / 2$ & $1 / 2$ \\
\hline
\end{tabular}

Table 2. Additive quantum numbers at an infinite sublayer level. All quantum number is just one-half.

\begin{tabular}{ccccccc}
\hline & \multicolumn{2}{c}{ First generation } & Second generation & \multicolumn{2}{c}{ Third generation } \\
\hline Flavors at an infinite sublayer level & $d(\infty)$ & $u(\infty)$ & $s(\infty)$ & $c(\infty)$ & $b(\infty)$ & $t(\infty)$ \\
$N \rightarrow \infty$ & 0 & 0 & 0 & 0 & 0 & 0 \\
Baryon number $B$ & $-1 / 2$ & $1 / 2$ & $-1 / 2$ & $1 / 2$ & $-1 / 2$ & $1 / 2$ \\
Electric charge $Q$ & $1 / 2$ & $1 / 2$ & 0 & 0 & 0 & 0 \\
Isospin $I$ & $-1 / 2$ & $1 / 2$ & 0 & 0 & 0 & 0 \\
Third component of isospin $I_{3}$ & 0 & 0 & $-1 / 2$ & 0 & 0 & 0 \\
Strangeness number spin $S / 2$ & 0 & 0 & 0 & $1 / 2$ & 0 & 0 \\
Charm number spin $C / 2$ & 0 & 0 & 0 & 0 & $-1 / 2$ & 0 \\
Bottomness number spin $\mathcal{B} / 2$ & 0 & 0 & 0 & 0 & 0 & $1 / 2$ \\
Topness number spin $T / 2$ & $-1 / 2$ & $1 / 2$ & $-1 / 2$ & $1 / 2$ & $-1 / 2$ & $1 / 2$ \\
\hline Third component of weak isospin & $\left(t_{3}\right)_{L}$ & & & & & \\
\hline
\end{tabular}

Now, we compare our prediction value $R=15 / 4=3.75$ with the measurements in electron-positron annihilation into muon pairs and quark pairs.

In a previous paper [16] we compared the ratio $R$ with the data for the entire PETRA energy region [17].

Here, the data come from many storage-ring collider experiments [18]-[25].

The predicted 5 quark value $R=15 / 4=3.75$ is shown by the solid straight line in Figure 5 and agrees with the experiments.

\section{CP Violation in the Doublets of Quarks $u_{\infty}$ and}

\section{Antiquarks $u_{\infty}^{C P}$}

In our model, the left-handed weak isospin doublet of quarks and right-handed singlet are written as: 


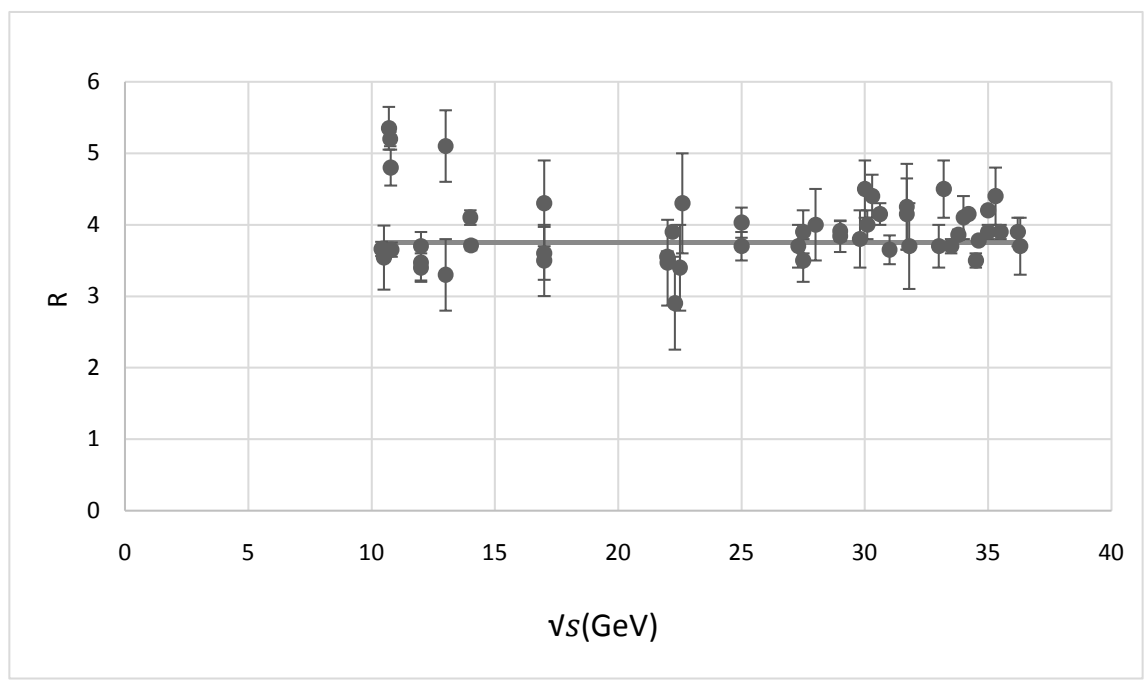

Figure 5. Various experimental values $R$ [18]-[25] versus predicted value $R=3.75$.

$$
\left(\begin{array}{c}
u_{\infty L} \\
u_{\infty L}^{c p}
\end{array}\right), u_{\infty R}, u_{\infty R}^{c p}
$$

The symmetry between the left-handed and right-handed quarks is broken. To explain CP violation, we introduce a phase factor $A=\mathrm{e}^{i \delta}$ with an ordinary number $\delta$ into the left-handed weak isospin doublet, viz.

$$
\left(\begin{array}{c}
u_{\infty L} \\
A u_{\infty L}^{c p}
\end{array}\right), u_{\infty R}, u_{\infty R}^{c p}
$$

The Lagrangian describing the electroweak interactions of $u_{\infty}$ and $u_{\infty}^{C P}$ quarks is written as follows:

$$
\begin{aligned}
L= & -\frac{1}{4} \mathbf{W}_{\mu v} \cdot \mathbf{W}^{\mu v}-\frac{1}{4} B_{\mu \nu} B^{\mu v}+\bar{\chi}_{L} \gamma^{\mu}\left(i \partial_{\mu}-\frac{g}{2} \tau \cdot \mathbf{W}_{\mu}-g^{\prime} \frac{Y}{2} B_{\mu}\right) \chi_{L} \\
& +\bar{u}_{\infty R} \gamma^{\mu}\left(i \partial_{\mu}-g^{\prime} \frac{Y}{2} B_{\mu}\right) u_{\infty R}+\overline{u_{\infty R}^{c p}} \gamma_{\mu}\left(i \partial_{\mu}-g^{\prime} \frac{Y}{2} B_{\mu}\right) u_{\infty R} \\
& +\left|\left(i \partial_{\mu}-\frac{g}{2} \tau \cdot \mathbf{W}_{\mu}-g^{\prime} \frac{Y}{2} B_{\mu}\right) \Phi\right|^{2}-V(\Phi) \\
& -\left(g_{u_{\infty}^{c p}} \overline{\tilde{\chi}}_{L} \Phi u_{\infty}^{c p}+g_{u_{\infty}} \bar{\chi}_{L} \tilde{\Phi} u_{\infty R}+\text { H.c. }\right)
\end{aligned}
$$

where $g$ and $g^{\prime}$ are coupling constants of $S U(2)$ and $U(1)$, respectively, $\mathbf{W}_{\mu}$ are three gauge fields of $S U(2), B_{\mu}$ is the gauge field of $U(1), \frac{\tau}{2}$ are the generators of $S U(2)$ and $Y$ is the weak hypercharge. Furthermore, $g_{u_{\infty}^{c p}}$ and $g_{u_{\infty}}$ are coupling constants of $u_{\infty}^{c p}$ and $u_{\infty}$ quarks with Higgs boson. $\Phi$ is the Higgs doublet and $\tilde{\Phi}$ is its complex representation.

To account for $C P$ violation, we shall consider the quantum numbers of weak isospin $t_{3}=1 / 2$ and $t_{3}=-1 / 2$ for the ultimate particles $u_{\infty L}$ and $u_{\infty L}^{C P}$, where $u_{\infty L}^{C P}$ means the left-handed particle operated upon by charge conjugation $C$ and then by parity $P$, viz., 


$$
u_{\infty L}^{C P} \equiv \gamma^{0} C \gamma^{0} \frac{1}{2}\left(1-\gamma_{5}\right) u_{\infty}^{*}
$$

At the infinite sublayer quark model, the hepercharge of $u_{\infty L}$ and $u_{\infty L}^{C P}$ quarks becomes zero by applying the Gell-Mann-Nishijima relation to weak quantum numbers.

Now we consider the doublet $\chi_{L}=\left(u_{\infty L} A u_{\infty L}^{C P}\right)^{\mathrm{T}}$ where the superscript $\mathrm{T}$ means transposed. Then the Lagrangian describing the electroweak interactions is written as follows:

$$
L=\bar{\chi}_{L} \gamma^{\mu}\left(i \partial_{\mu}-\frac{g}{2} \tau \cdot \mathbf{W}_{\mu}\right) \chi_{L}
$$

where $g$ is the coupling constant of $S U(2)$ and $U(1), \mathbf{W}_{\mu}$ are three gauge fields of $S U(2)$ and $\frac{\tau}{2}$ are the generators of $S U(2)$.

In the electroweak theory, $S U(2) \times U(1)$ is adopted as a gauge group from the Lagrangian $L$ in Equation (13).

Now we consider the doublet $\chi_{L}=\left(u_{\infty L} A u_{\infty L}^{C P}\right)^{\mathrm{T}}$ where the superscript $\mathrm{T}$ means transposed. If $A$ is a scalar, then $C P$ is not violated. To account for $C P$ violation, it is necessary to extend a scalar phase factor $A$ to a matrix formed by $\gamma$ matrix.

If $A$ is a scalar, for example, $A=\mathrm{e}^{i \delta}$, where the phase $\delta$ is an ordinary number.

Then, $\chi_{L}$ is written as

$$
\chi_{L}=\left(\begin{array}{c}
u_{\infty L} \\
\mathrm{e}^{i \delta} u_{\infty L}^{c p}
\end{array}\right) .
$$

It is important to note that the phase factor $\mathrm{e}^{i \delta}$ cannot be eliminated by redefining the phase. Then we considered the internal structure which is described by the $S U(2)$ noncommutative geometry. That is, the internal space operated by $S U(2)$ is called the representation space mathematically. This is a two-dimensional vector space over the complex Körper C. Here we deform this space and assume a plane with the periodical boundary in each coordinate direction. After all, the internal space is described by the deformed $S U(2)$-bundle, in which the fiber associate in time-space is torus. Thus we consider the periodical condition on a two-dimensional vector space. By imposing the noncommutative conditions on two periodical functions on torus, we consider CP violation from the noncommutative internal structure on such space.

Let the spinor components of quarks $u_{\infty}$ and $u_{\infty}^{c p}$ be $u_{\infty \alpha}$ and $u_{\infty \beta}^{c p}$. These are the coordinates in the noncommutative internal structure. Thus we obtain

$$
u_{\infty \alpha}^{*} u_{\infty \beta}^{c p}+u_{\infty \beta}^{c p} u_{\infty \alpha}^{*}=k_{\alpha \beta} .
$$

where $u^{*}$ is the complex conjugate of $u$ and $k_{\alpha \beta}$ is a non-zero c-number. The plus sign came from the fact that $u_{\alpha}$ and $d_{\beta}$ are Grassmann numbers. If we change the phase of the first isospin component $u_{\alpha}$ in Equation (4) and redefine $u_{\alpha}^{\prime} \equiv \mathrm{e}^{i \delta} u_{\alpha}$, the constant $k_{\alpha \beta}$ in Equation (15) should change. This is not allowed. Therefore, CP is violated in the doublet of $u_{\infty L}$ and $u_{\infty L}^{C P}$ quarks in (9). 
Now we consider the doublet $\chi_{L}=\left(u_{\infty L} A u_{\infty L}^{C P}\right)^{\mathrm{T}}$, where the superscript T means transposed. To satisfy the $S U(2)_{L}$ gauge symmetry, the matrix $A$ must satisfy the following condition:

$$
A C A^{*} C=-I_{4}
$$

where $I_{4}$ is a $4 \times 4$ unit matrix. For example, the matrix $A$ is written follows:

$$
A=\gamma_{5} \gamma^{0} \exp \left[i d_{\mu} \gamma^{\mu}\right]
$$

where $d_{\mu}$ is a real vector in Minkowski space and independent of space.

Thus, we can show $C P$ violation in the doublet $\chi_{L}=\left(u_{\infty L} A u_{\infty L}^{C P}\right)^{\mathrm{T}}$ at an infinite sublayer quark model [26] [27] [28] [29] [30]. We applied Equation (17) to $\mathrm{CP}$ violation in $\beta$-decay by considering the preon $a_{L}$ and $a_{L}^{C P}$ where $a_{L}=u_{\infty L}$ and $a_{L}^{C P}=u_{\infty L}^{C P}$ in these references [26] [27] [28] [29] [30] and showed theself-energy removal and anomaly freedom.

\section{Leptons and Gauge Bosons Composed of Quarks $u_{\infty}$ and Anti-Quarks $u_{\infty}^{C P}$}

Within the framework of electroweak theory, a quark current $\bar{u}_{N} \gamma_{\mu}\left[\left(1-\gamma_{5}\right) / 2\right] d_{N}$ at a quark level $\mathrm{N}$ to the weak vector bosons $W^{-}$and $W^{+}$as

$$
\left(d_{N}\right)_{L} \rightarrow\left(u_{N}\right)_{L}+W^{-}
$$

or

$$
\left(d_{N}\right)_{L}+W^{+} \rightarrow\left(u_{N}\right)_{L}
$$

This is shown in Figure 6

From the infinite sub-layer quark model at $N=\infty$, the above equation is a consequence of the following process:

$$
\begin{aligned}
& \left(d_{\infty}\right)_{L} \rightarrow\left(u_{\infty}\right)_{L}+W^{-} \\
& \left(d_{\infty}\right)_{L}+W^{+} \rightarrow\left(u_{\infty}\right)_{L}
\end{aligned}
$$

The ultimate quark $u_{\infty}$ and anti-quark $d_{\infty}=\bar{u}_{\infty}=u_{\infty}^{C P}$ are structureless. Therefore, if the gauge bosons $W^{-}$and $W^{+}$are composite particles, then we obtain $W^{-}=\left(u_{\infty\llcorner}^{C P} u_{\infty L}^{C P}\right)$ and $W^{+}=\left(u_{\infty L} u_{\infty L}\right)$. From the process

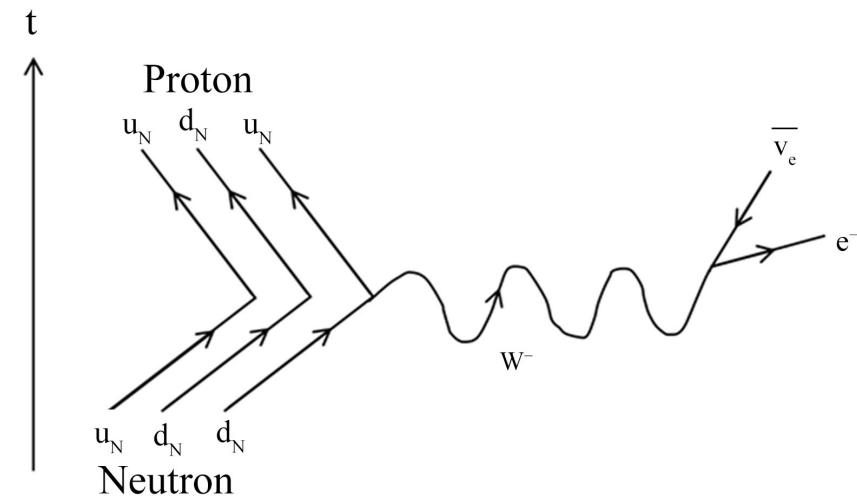

Figure 6. $\left(d_{N}\right)_{L} \rightarrow\left(u_{N}\right)_{L}+W^{-}$and $W^{-}+\bar{v}_{e L} \rightarrow e_{L}^{-}$graph. 


$$
e_{L}^{+} e_{L}^{-} \rightarrow W^{+} W^{-}
$$

which is mediated by the gauge boson $Z^{0}$, we obtain $Z^{0}=\left(u_{\infty L} u_{\infty L}^{C P}\right)$.

Thus the gauge bosons are composed of the ultimate particles $u_{\infty}$ and $u_{\infty}^{C P}$ at an infinite sub-layer quark level as follows:

$$
W^{-}=\left(u_{\infty L}^{C P} u_{\infty L}^{C P}\right), \quad W^{+}=\left(u_{\infty L} u_{\infty L}\right), Z^{0}=\left(u_{\infty L} u_{\infty L}^{C P}\right)
$$

\section{Composite Model of Leptons from the Ultimate Particles} $u_{\infty}$ and $u_{\infty}^{C P}$

The electron $e^{-}$and the electron-neutrino $v_{e}$ are the first generation leptons.

If the electron is a composite particle and the neutrino is structureless, we can construct as

$$
e_{L}^{-}=\left(u_{\infty L}^{C P} u_{\infty L}^{C P} \overline{\bar{v}}_{e L}\right) \text { and } v_{e L}=\left(u_{\infty L} u_{\infty\llcorner}^{C P} a_{L}\right)
$$

from $W^{-}+\bar{v}_{e L} \rightarrow e_{L}^{-}$in Figure 6. Here aL is a chargeless particle with spin $1 / 2$.

The muon $\mu^{-}$and the muon-neutrino $v_{\mu}$ are the second generation leptons.

We can construct as

$$
\mu_{L}^{-}=\left(u_{\infty L}^{C P} u_{\infty L}^{C P} v_{\mu}\right) \text { and } v_{\mu L}=\left(u_{\infty L} u_{\infty L}^{C P} a_{L}\right)
$$

from the decay

$$
\mu^{-} \rightarrow W^{-}+v_{\mu} .
$$

The tau $\tau^{-}$and the tau-neutrino $v_{\tau}$ are the third generation leptons.

We can construct as

$$
\tau_{L}^{-}=\left(u_{\infty L}^{C P} u_{\infty L}^{C P} v_{\tau}\right) \text { and } v_{\tau L}=\left(u_{\infty L} u_{\infty L}^{C P} a_{L}\right)
$$

from the decay

$$
\tau^{-} \rightarrow W^{-}+v_{\tau} .
$$

Thus, the electron, muon, tau and the corresponding neutrinos are composite particles.

\section{Four Generations Composed of Quarks $u_{\infty}$ and

$$
\text { Anti-Quarks } \boldsymbol{u}_{\infty}^{C P}
$$

In the standard model, we cannot give any limitations to the members of generations of quarks and leptons.

We can restrict the number of neutrinos to four from astrophysical considerations [31]. Now we can derive

The number of generations by applying the $C$ (Charge), $P$ (Parity), $T$ (Time) transformation and the $S U(2)_{L} \times U(1)$ gauge theory to the $u_{\infty}$ and anti-quarks $u_{\infty}^{C P}$. Thus we obtain

$$
\left(u_{\infty L}^{C P} u_{\infty L}\right)^{T}\left(u_{\infty L}^{P} u_{\infty L}^{C}\right)^{T}\left(u_{\infty L}^{T} u_{\infty L}^{C P T}\right)^{T}\left(u_{\infty L}^{T P} u_{\infty L}^{C T}\right)^{T}
$$




$$
\begin{aligned}
& \left(u_{\infty L}^{C T} u_{\infty L}\right)^{T}\left(u_{\infty L}^{T} u_{\infty L}^{C}\right)^{T}\left(u_{\infty L}^{P} u_{\infty L}^{C P T}\right)^{T}\left(u_{\infty L}^{T P} u_{\infty L}^{C P}\right)^{T} \\
& \left(u_{\infty R}^{C P} u_{\infty R}\right)^{T}\left(u_{\infty R}^{P} u_{\infty R}^{C}\right)^{T}\left(u_{\infty R}^{T} u_{\infty R}^{C P T}\right)^{T}\left(u_{\infty R}^{T P} u_{\infty R}^{C T}\right)^{T} \\
& \left(u_{\infty R}^{C T} u_{\infty R}\right)^{T}\left(u_{\infty R}^{T} u_{\infty \circ}^{C}\right)^{T}\left(u_{\infty R}^{P} u_{\infty R}^{C P T}\right)^{T}\left(u_{\infty R}^{T P} u_{\infty R}^{C P}\right)^{T}
\end{aligned}
$$

From these 16 doublets under $S U(2)$, we can choose the following four doublets, which are invariant under $S U(2)_{L} \times U(1)$ gauge transformation:

$$
\left(\begin{array}{c}
u_{\infty L} \\
u_{\infty L}^{C P}
\end{array}\right)\left(\begin{array}{c}
u_{\infty L}^{T} \\
u_{\infty L}^{C P T}
\end{array}\right)\left(\begin{array}{c}
u_{\infty R}^{P} \\
u_{\infty R}^{C}
\end{array}\right)\left(\begin{array}{c}
u_{\infty R}^{T P} \\
\left(u_{\infty \circ}^{C T}\right.
\end{array}\right)
$$

We have already derived four generations in the preons model [32]. It is natural to assume that the second, third and fourth generations are the excited states of the first generation. Therefore, quark $u_{\infty}$ and anti-quarks $u_{\infty}^{C P}$ of the first generation are regarded as the ultimate particles in the universe.

\section{Higgs Bosons Composed of Ultimate Quarks $u_{\infty}$ and $\boldsymbol{u}_{\infty}^{C P}$}

The present universe is full of dark matter candidate particles $u_{\infty}$ and $u_{\infty}^{C P}$. Here we will show that the Higgs bosons are composed of dark matter candidate particles $u_{\infty L}$ and $u_{\infty L}^{C P}$ quarks.

In the following, we shall consider the composite model of the Higgs bosons. Consider the weak isospin doublet $\chi_{L}=\left(u_{\infty L} u_{\infty L}^{C P}\right)^{T}$ in the $S U(2)_{L} \times U(1)$ gauge transformation. Then, the Lagrangian is invariant under the following infinitesimal gauge transformation:

$$
\chi^{\prime}=\left(1+i \frac{g}{2} \alpha \cdot \tau\right) \chi
$$

where $g$ is the coupling constant, $\alpha$ are parameters in $S U(2)_{L}$ and $\tau / 2$ are generators of $S U(2)_{L}$. It was shown that the weak isospin doublet $\left(u_{\infty L} u_{\infty L}^{C P}\right)^{T}$ does not give any limitations to the parameters $\alpha$. Here the quark $u_{\infty L}$ has quantum numbers of weak isospin $t=1 / 2$, third component of weak isospin $t_{3}=1 / 2$, hypercharge $Y=0$ and electric charge $Q=\frac{1}{2}|e|$ while the $u_{\infty L}^{C P}$ quark has quantum numbers of $t=1 / 2, t_{3}=-1 / 2, Y=0$ and $Q=-\frac{1}{2}|e|$.

Now consider the right-handed quark $u_{\infty R}$ with quantum numbers of $t=0, t_{3}=0, Y=1$ and $Q=\frac{1}{2}|e|$. Under the infinitesimal gauge transformation, we obtain

$$
\begin{aligned}
& u_{\infty R}^{\prime}=\left(1+i \frac{g^{\prime}}{2} \beta\right) u_{\infty R} \\
& \overline{u_{\infty R}^{\prime}}=\overline{u_{\infty}}\left(1-i \frac{g^{\prime}}{2} \beta\right)
\end{aligned}
$$

where $g^{\prime}$ is the coupling constant of $U(1)$ and $\beta$ is the parameter in $U(1)$. 
From $u_{\infty L}$ and $u_{\infty R}$ quarks, we construct the Higgs scalar $\phi$ of $S U(2)_{L} \times U(1)$ symmetry as follows:

$$
\phi=\left(\begin{array}{ll}
\overline{u_{\infty R}} & u_{\infty L} \\
\overline{u_{\infty R}} & u_{\infty L}^{C P}
\end{array}\right)
$$

where the electric charge of $\left(\overline{u_{\infty R}} u_{\infty L}\right)$ is 0 and the electric charge of $\left(\overline{u_{\infty R}} u_{\infty L}^{C P}\right)$ is $-|e|$. Under the gauge transformation of $\chi$ and $u_{\infty R}$ in Equations (24) and (25), the Higgs scalar $\phi$ transforms as

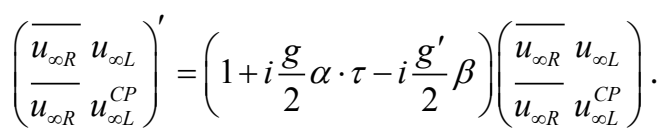

This corresponds to the $S U(2)_{L} \times U(1)$ gauge transformation of the field with $t=1 / 2$ and $Y=-1$. Therefore, the Lagrangian describing the isospin doublet, which is constructed from $u_{\infty L L}$ and $u_{\infty R}$ quarks, is invariant under the $S U(2)_{L} \times U(1)$ gauge transformation.

In the following, we shall consider the following Higgs potential $V(\phi)$ to give the mass to gauge bosons and leptons:

$$
V(\phi)=\mu^{2} \phi^{\dagger} \phi+\lambda\left(\phi^{\dagger} \phi\right)^{2}
$$

where $\mu^{2}$ is the mass parameter and $\lambda$ is the coupling constant. $\mu^{2}<0$ and $\lambda>0$. Then, $V(\phi)$ becomes a minimum when

$$
\phi^{\dagger} \phi=\left|\overline{u_{\infty R}} u_{\infty L}\right|^{2}+\left|\overline{u_{\infty R}} u_{\infty L}^{C P}\right|=-\frac{\mu^{2}}{2 \lambda} .
$$

Putting

$$
\mathrm{v}^{2}=-\frac{\mu^{2}}{\lambda}=2\left(\left|\overline{u_{\infty R}} u_{\infty L}\right|+\left|\overline{u_{\infty R}} u_{\infty L}^{C P}\right|^{2}\right)
$$

and defining the vacuum expectation value $\phi_{0}$ of $\phi$ as

$$
\phi_{0}=\frac{1}{\sqrt{2}}\left(\begin{array}{l}
\mathrm{v} \\
0
\end{array}\right)
$$

The Higgs potential produces the spontaneous symmetry breakdown as well as the usual standard model. In this case, quantum numbers of $\phi_{0}$ have $t=1 / 2, t_{3}=1 / 2$ and $Y=-1$, so that the electric charge $Q=0$.

Define the Higgs field $\phi$ by

$$
\phi=\frac{1}{\sqrt{2}}\left(\begin{array}{c}
\mathrm{v}+h \\
0
\end{array}\right)
$$

where $h$ is the piece over and above the vacuum. Thus, the vacuum expectation value in Equations (32) and (33) gives the masses to gauge bosons, quarks and leptons.

\section{Einstein's Cosmological Constant Is Derived from the Higgs Potential}

Einstein, in his heuristic derivation, introduced a cosmological constant $\Lambda$ into 
his field equations [33].

$$
R_{\mu v}-\frac{1}{2} \mathrm{~g}_{\mu \nu} R+\Lambda \mathrm{g}_{\mu v}=\kappa T_{\mu v}
$$

where $R_{\mu v}$ is the Ricci tensor, $\mathrm{g}_{\mu v}$ the metric tensor, $R$ the scalar curvature, $\kappa$ Einstein's gravitational constant and $T_{\mu \nu}$ the stress-energy tensor.

The constant of $\kappa$ is found to have a value of

$$
\kappa=\frac{8 \pi G}{c^{4}}
$$

where $c$ is the speed of light and $G$ Newton's gravitational constant.

Here we summarize the Higgs mechanism using natural units of $c=\hbar=1$.

The Lagrangian describing the electroweak interactions may be written in the form

$$
L_{e w}=-\frac{1}{4} \mathbf{W}_{\mu v} \cdot \mathbf{W}^{\mu v}-\frac{1}{4} B_{\mu v} B^{\mu \nu}+\left|\left(i \partial_{\mu}-\frac{\alpha}{2} \tau \cdot \mathbf{W}_{\mu}-\frac{\alpha^{\prime}}{2} B_{\mu}\right) \varphi\right|^{2}-V(\varphi),
$$

where $\alpha$ and $\alpha^{\prime}$ are coupling constants of $S U(2)$ and $U(1)$, respectively, $\mathbf{W}_{\mu}$ are three gauge fields of $S U(2), B_{\mu}$ is the gauge field of $U(1), \frac{\tau}{2}$ are the generators of $S U(2)$. We omitted the leptons and quarks parts and described only the kinetic terms of gauge bosons and Higgs fields. The Higgs potential $V(\varphi)$ is written as

$$
V(\varphi)=\mu^{2}|\varphi|^{2}+\rho|\varphi|^{4} .
$$

where $\mu^{2}$ is the mass parameter and $\rho$ is the coupling constant. Furthermore, $\mathbf{W}_{\mu v}$ and $B_{\mu \nu}$ are defined as

$$
\mathbf{W}_{\mu v}=\partial_{\mu} \mathbf{W}_{v}-\partial_{v} \mathbf{W}_{\mu}-\alpha \mathbf{W}_{\mu} \times \mathbf{W}_{v}, \quad B_{\mu v}=\partial_{\mu} B_{v}-\partial_{v} B_{\mu} .
$$

To consider the Higgs mechanism, the conditions of $\mu^{2}<0$ and $\rho>0$ are necessary. Then the Higgs potential $V(\varphi)$ has a graph which looks like a champagne bottle and the minimum energy value is not at $\varphi=0$. Defining the vacuum expectation value of $\varphi$ as

$$
\langle 0|\varphi(x)| 0\rangle=\frac{1}{\sqrt{2}}\left(\begin{array}{l}
0 \\
\mathrm{v}
\end{array}\right), \quad \mathrm{v}=\sqrt{-\frac{\mu^{2}}{\rho}} .
$$

then $V(\varphi)$ becomes a minimum. Putting (39) into the third term on the right hand side of (38), we obtain

$$
\begin{aligned}
& \left|\left(i \partial_{\mu}-\frac{\alpha}{2} \tau \cdot \mathbf{W}_{\mu}-\frac{\alpha^{\prime}}{2} B_{\mu}\right) \varphi\right|^{2} \\
& =\frac{1}{8} \mid\left(\begin{array}{cc}
\alpha W_{\mu}^{3}+\alpha^{\prime} B_{\mu} & \alpha\left(W_{\mu}^{1}-i W_{\mu}^{2}\right) \\
\alpha\left(W_{\mu}^{1}+i W_{\mu}^{2}\right) & -\alpha W_{\mu}^{3}+\alpha^{\prime} B_{\mu}
\end{array}\right)\left(\left.\begin{array}{l}
0 \\
\mathrm{v}
\end{array}\right|^{2}\right. \\
& =\left(\frac{1}{2} \mathrm{v} \alpha\right)^{2} W_{\mu}^{+} W^{-\mu}+\frac{1}{8} \mathrm{v}^{2}\left(\alpha W_{\mu}^{3}-\alpha^{\prime} B_{\mu}\right)^{2}+0\left(\alpha^{\prime} W_{\mu}^{3}+\alpha B_{\mu}\right)^{2} \\
& =M_{W}^{2} W_{\mu}^{+} W^{-\mu}+\frac{1}{2} M_{Z}^{2} Z_{\mu}^{2}+\frac{1}{2} M_{A}^{2} A_{\mu}^{2} \text {. }
\end{aligned}
$$


Here we normalize the gauge field as

$$
W_{\mu}^{ \pm}=\frac{1}{\sqrt{2}}\left(W_{\mu}^{1} \mp i W_{\mu}^{2}\right), Z_{\mu}=\frac{\alpha W_{\mu}^{3}-\alpha^{\prime} B_{\mu}}{\sqrt{\alpha^{2}+\alpha^{\prime 2}}}, A_{\mu}=\frac{\alpha^{\prime} W_{\mu}^{3}+\alpha B_{\mu}}{\sqrt{\alpha^{2}+\alpha^{\prime 2}}} .
$$

From (40), we obtain the massive $W$ bosons which have mass $M_{W}$, massive $Z$ boson mass $M_{Z}$ and photon mass $M_{A}$ as

$$
M_{W}=\frac{1}{2} \mathrm{v} \alpha, \quad M_{Z}=\frac{1}{2} \mathrm{v} \sqrt{\alpha^{2}+\alpha^{\prime 2}}, \quad M_{A}=0
$$

In the following, we shall derive the cosmological constant by considering the gravitational potential instead of the Higgs potential.

Consider a general space-time manifold with metric $\mathrm{g}_{\mu v}(x)$. The determinant of $g_{\mu v}(x)$ is defined as

$$
g(x) \equiv \operatorname{det}_{\mu v}(x) .
$$

Furthermore, we shall consider the space-time dependent field $\varphi_{G}(\mathrm{~g}(x))$ via $(\sqrt{-g(x)})^{\frac{1}{2}}$. To match with the electroweak theory, we construct $\varphi_{G}(g(x))$ from $S U(2)$ doublet in the form

$$
\varphi_{G}(\mathrm{~g}(x))=\frac{1}{\sqrt{2}}\left(\begin{array}{c}
\varphi_{1}(\mathrm{~g})+i \varphi_{2}(\mathrm{~g}) \\
\mathrm{v}+i \varphi_{3}(\mathrm{~g})
\end{array}\right) .
$$

Now we shall consider the following gravitational potential $V\left(\varphi_{G}\right)$ :

$$
V\left(\varphi_{G}\right)=\mu^{2}\left|\varphi_{G}\right|^{2}+\rho\left|\varphi_{G}\right|^{4} .
$$

Then, the vacuum is defined as a minimum point of $V\left(\varphi_{G}\right)$.

The Lagrangian $L_{e w}$ in (38) is rewritten as $L_{e w}\left(\varphi_{G}\right)$ by replacing $V(\varphi)$ by $V\left(\varphi_{G}\right)$ and $\varphi$ by $\varphi_{G}$. The Hilbert-Einstein Lagrangian in empty space $\left(T_{\mu \nu}=0\right)$ is written in the form

$$
L_{H E}=\frac{1}{2 \kappa} \sqrt{-\mathrm{g}} R .
$$

Then, we obtain the total Lagrangian as

$$
L=L_{H E}+L_{e w}\left(\varphi_{G}\right) .
$$

It should be noted that after spontaneous symmetry is broken, the differential derivative of $\varphi_{G}$ should be replaced by the covariant derivative. Now we fix gauge properly by using three degrees freedom $S U(2)$ gauge invariance. Then we can eliminate the three fields, $\varphi_{1}, \varphi_{2}$ and $\varphi_{3}$ appearing in (46). Thus, we can write the vacuum expectation value as

$$
\varphi_{G 0}=\left\langle 0\left|\varphi_{G}(\mathrm{~g}(x))\right| 0\right\rangle=\frac{1}{\sqrt{2}}\left(\begin{array}{l}
0 \\
\mathrm{v}
\end{array}\right) .
$$

From this, we obtain

$$
\left|\varphi_{G 0}\right|^{2}=\frac{1}{2} v^{2}
$$


where $\mathrm{v}=\sqrt{-\frac{\mu^{2}}{\rho}}$.

Thus, we can give the masses to the gauge bosons from the same way in Equations (41)-(44).

Now, $\varphi_{G}$ is expanded around $\varphi_{G 0}$ and we can write

$$
\left|\varphi_{G}\right|^{2}=\frac{1}{2} v^{2}+\lambda(\sqrt{-g})^{\frac{1}{2}}
$$

where $\lambda$ is an expansion coefficient and has a real number value. Substituting (52) into (49), we shall consider only the terms contributing to the gravitational field equations. We don't consider the term giving the masses to $\mathrm{W}$ bosons, $\mathrm{Z}$ boson and photon, and the interactions terms.

The gravitational potential $V\left(\varphi_{G}\right)$ is written as

$$
\begin{aligned}
V\left(\varphi_{G}\right) & =\mu^{2}\left|\varphi_{G}\right|^{2}+\rho\left|\varphi_{G}\right|^{4} \\
& =\frac{\left(2 \mu^{2}+\rho \mathrm{v}^{2}\right) \mathrm{v}^{2}}{4}+\left(\mu^{2}+\rho \mathrm{v}^{2}\right) \lambda(\sqrt{-\mathrm{g}})^{\frac{1}{2}}+\rho \lambda^{2} \sqrt{-\mathrm{g}} \\
& =-\frac{1}{4} \rho \mathrm{v}^{4}+\rho \lambda^{2} \sqrt{-\mathrm{g}} .
\end{aligned}
$$

Here, the first order term of $\lambda$ vanishes, since $\mu^{2}+\rho v^{2}=0$. Furthermore, $\varphi_{G}$ is expanded from (52) as

$$
\varphi_{G}=\frac{1}{\sqrt{2}}\left(\begin{array}{c}
0 \\
v+\frac{1}{v} \lambda(\sqrt{-g})^{\frac{1}{2}}-\frac{1}{2 v^{3}} \lambda^{2} \sqrt{-g}+\frac{1}{2 v^{5}} \lambda^{3}(\sqrt{-g})^{\frac{3}{2}}-\cdots
\end{array}\right) .
$$

The constant terms vanish at $\partial_{\mu} \varphi_{G}$ and also $\partial_{\mu} \sqrt{-g}$ is included in every terms except the constant terms. If we consider the gravitational field, the covariant derivative of a scalar density $\sqrt{-g}$ is defined by the relation

$$
\nabla_{\mu} \sqrt{-\mathrm{g}}=\partial_{\mu} \sqrt{-\mathrm{g}}-\Gamma_{\alpha \mu}^{\alpha} \sqrt{-\mathrm{g}}
$$

where $\Gamma_{\mu v}^{\alpha}$ is the Levi-Civita connection. From direct calculations, we get

$$
\nabla_{\mu} \sqrt{-\mathrm{g}}=0 .
$$

This is obtained from the relation $\partial_{\mu} \sqrt{-g}=\sqrt{-g} \Gamma_{\alpha \mu}^{\alpha}$. Thus, it is easily seen that the term $\left|i \partial_{\mu} \varphi_{G}\right|^{2}$ in the Lagrangian becomes zero. Finally, we obtain the following Lagrangian after spontaneous symmetry is broken,

$$
L=\frac{1}{2 \kappa} \sqrt{-\mathrm{g}} R+\frac{\rho \mathrm{v}^{2}}{4}-\rho \lambda^{2} \sqrt{-\mathrm{g}} .
$$

Suppose that the action of the gravitational field equation is given by

$$
I_{G}=\int \mathrm{d} x^{4} L .
$$

The action principle then tells us that the variation of this action with respect to the metric $g^{\mu v}$ is zero, yielding

$$
\delta I_{G}=\int \mathrm{d} x^{4}\left(\frac{1}{2 \kappa^{2}} \sqrt{-\mathrm{g}} G_{\mu \nu} \delta \mathrm{g}^{\mu \nu}+\frac{\rho \lambda^{2}}{2} \sqrt{-\mathrm{g}} \mathrm{g}_{\mu \nu} \delta \mathrm{g}^{\mu \nu}\right)=0
$$


where $G_{\mu \nu}$ is the Einstein tensor in the form

$$
G_{\mu v}=R_{\mu v}-\frac{1}{2} \mathrm{~g}_{\mu v} R .
$$

Finally we obtain the gravitational field equation in empty space

$$
R_{\mu v}-\frac{1}{2} \mathrm{~g}_{\mu v} R+\rho \kappa \lambda^{2} \mathrm{~g}_{\mu v}=0 .
$$

Compared to (36), we obtain the cosmological constant

$$
\Lambda=\rho \kappa \lambda^{2} .
$$

Thus, we can derive the cosmological constant from spontaneous symmetry breaking.

\section{Conclusions and Discussions}

It is shown that there exists an infinite number of ultimate quarks $u_{\infty}$ and $u_{\infty}^{C P}$ as the ultimate building blocks of the universe. This is derived from both an infinite sub-layer quark model and the Cantor set model. The existence of $u_{\infty}$ and $u_{\infty}^{C P}$ quarks is confirmed by the electron-positron experiments in high energy physics.

It is also shown that $\mathrm{CP}$ is violated in only one generation to account for asymmetry of particles and anti-particles. The second, third and fourth generations are assumed to be the excitated states of the first generation. The fourth generation is derived from the $C P T$ transformation and the $S U(2)_{L} \times U(1)$ gaugetheory.

Leptons, quarks, gauge bosons and Higgs bosons are composed of $u_{\infty}$ and $u_{\infty}^{C P}$ quarks. It is also shown that the Einstein's cosmological constant is derived from the Higgs potential via spontaneous symmetry breaking and the masses are produced. Einstein, in his heuristic derivation, introduced a cosmological constant into his field equations to construct a staticmodel of the universe. From the modern cosmology, the positive cosmological constant indicates that the universe is expanding at an accelerating rate; further possibilities exist under the general heading dark matter and dark energy. Thus, recently, there has been great interest in dark matter, dark energy and gravitational waves.

For example, these problems are discussed in the framework of the extended gravity theories [34]. The dark matter problem is also discussed inhypershere world-universe model [35].

Especially, it is interesting to note that the magic and mysterious fine structure constant $1 / \alpha=137$ was derived [36].

We proposed the possibility of generating the gravitational waves under the terrestrial conditions [37].

Dark matter is usually classified into two categories: baryonic and nonbaryonic dark matter. The composition of baryonic dark matter is considered to be black holes, neutron stars, white dwarfs, very faint stars, and cloud of nonluminous gas. 
However, if most of universe is made up of baryons, we encounter serious contradiction in explaining the observed structure formation [38]. The non-baryonic dark matter is divided into hot dark matter moving rapidly and cold dark matter moving slowly. There are a number of ideas about the non-baryonic dark matter, for example, neutrinos, axions and neutralino.

The composition of the dark matter has not yet been discovered. We proposed that the ultimate quarrks $u_{\infty}$ and anti-quarks $u_{\infty}^{C P}$ are the cold dark matter candidates to comprise the universe, since they are absolutely stable and the non-baryonic particles with the baryon number zero.

Finally, it is concluded that a pair of an infinite number of $u_{\infty}$ quarks and $u_{\infty}^{C P}$ quarks is produced in the first moments after the Big Bang and form the nucleons, and leave approximately right relic abundance to account for the observed non-baryonic dark matter.

\section{Conflicts of Interest}

The authors declare no conflicts of interest regarding the publication of this paper.

\section{References}

[1] Sekine, M. (1985) The Structure of the Nucleon. International Journal of Theoretical Physics, 24, 701-705. https://doi.org/10.1007/BF00670877

[2] Griffiths, D. (2008) Introduction to Elementary Particles. Wiley-VCH, Weinheim, Germany.

[3] Sugita, K., Okamoto, Y. and Sekine, M. (2008) Dark Matter Candidate Particles, $C P$ Violation and Higgs Bosons. International Journal of Theoretical Physics, 47, 2875 2881. https://doi.org/10.1007/s10773-008-9720-3

[4] Sugita, K., Okamoto, Y. and Sekine, M. (2006) CP Violation via a Noncommutative SU(2)-Bundle Internal Structure. Physics Essays, 19, 55-58. https://doi.org/10.4006/1.3025780

[5] Sugita, K., Okamoto, Y. and Sekine, M. (2021) Differential Geometry for Theoretical Physics: From Commutative Geometry to Noncommutative Geometry. 5th Edition, Morikita Publishing Co. Ltd., Tokyo, 314-324. (in Japanese)

[6] Charlier, C. V. L. (1908) Eie Eine Unendliche Welt Augebaut Sein Kann. Arkiv Ror Mathematik Astronomi Och Fysik, vol, 15-40.

[7] Jaki, S.L (1969) The Paradox of Olbers' Paradox: A Case History of Scientific Thought. Herber and Herber, New York.

[8] Alfven, H. (1984) Cosmology: Myth or Science? Journal of Astrophysics and Astronomy, 5, 79-98. https://doi.org/10.1007/BF02714974

[9] Sekine, M. (2012) The Cantor Set Constructed from an Infinite Number of Quarks Constituting the Nucleon and the Dark Matter. Applied Physics Research, 4, 68-72. https://doi.org/10.5539/apr.v4n4p68

[10] Sugita, K., Okamoto, Y. and Sekine, M. (2011) Remarks on a Cosmological Constant by Spontaneous Symmetry Breaking. Physics Essays, 24, 136-138. https://doi.org/10.4006/1.3552879

[11] Okamoto, Y., Sugita, K. and Sekine, M. (1999) Weyl Transformation in Fermi Systems. Annalen der Physik, 8, 829-836. 
https://doi.org/10.1002/(SICI)1521-3889(199912)8:10<829::AID-ANDP829>3.0.CO; $\underline{2-\mathrm{L}}$

[12] Sugita, K., Okamoto, Y. and Sekine, M. (1998) Path Integrals and Quantum Electrodynamics. Morikita Publishing Co. Ltd., Tokyo, 193-216. (in Japanese)

[13] Cantor, G. (1883) Ueber unendliche, lineare Punktmannichfaltigkeiten. Mathematische Annalen, 21, 545-591. https://doi.org/10.1007/BF01446819

[14] Corda, C. (2014) Bohr-Like Model for Black Holes. AIP Conference Proceedings, 1648, Article ID: 020004.

[15] Gorishny, S.G., Kataev, A.L. and Larin, S.A. (1991) The O $\left(\alpha_{\mathrm{S}}^{3}\right)$ Corrections to $\sigma_{\text {tot }}(\mathrm{e}$ ${ }^{+} \mathrm{e}^{-} \rightarrow$ hadrons) and $\Gamma\left(\tau^{-} \rightarrow v_{\tau}+\right.$ hadrons) in QCD. Physics Letters, 259, 144-150. https://doi.org/10.1016/0370-2693(91)90149-K

[16] Sekine, M. (2021) Experimental Evidence of Non-Baryonic Dark Matter IH High Energy Physics. Journal of High Energy Physics, Gravitation and Cosmology, 7, 873-879. https://doi.org/10.4236/jhepgc.2021.73049

[17] Adeva, B., et al. (1986) Study of Hadron and Inclusive Muon Production from Annihilation at $39.79 \leq \sqrt{s} \leq 46.78 \mathrm{GeV}$. Physical Review, 34, 681-691.

[18] The CUSB Collaboration, Rice, E., et al. (1982) Search for Structure in $\sigma\left(e^{+} e^{-} \rightarrow\right.$ hadrons $)$ between $\sqrt{s}=10.34$ and $11.6 \mathrm{GeV}$. Physical Review Letters, 48, 906-909.

[19] The CELLO Collaboration, Behrend, H.J., et al. (1982) Measurement of the Reaction $e^{+} e^{-} \rightarrow \mu^{+} \mu^{-}$for $14 \leq \sqrt{s} \leq 36.4 \mathrm{GeV}$. Zeitschrift für Physik $C, 14,283-288$.

[20] The PLUTO Collaboration, Berger, C., et al. (1983) Measurement of the Muon Pair Asymmetry in $e^{+} e^{-}$Annihilation at $\sqrt{s}=34.7 \mathrm{GeV}$. Zeitschrift für Physik C, 21, 53-57. https://doi.org/10.1007/BF01648775

[21] The CLEO Collaboration, Giles, R. et al. (1984) Total Cross Section for ElectronPositron Annihilation into Hadron Final States in the Upsilon Energy Region Physical. Review, D29, 1285-1549.

[22] Adeva, B., et al. (1984) A Symmetry of Experimental Results from Mark J: High Energy $e^{+} e^{-}$Collisions at PETRA. Physics Report, 109, 131-226. https://doi.org/10.1016/0370-1573(84)90124-8

[23] The TASSO Collaboration, Althoff, M., et al. (1984) An Improved Measurement of Electroweak Coupling from $e^{+} e^{-} \rightarrow e^{+} e^{-}$and $e^{+} e^{-} \rightarrow \mu^{+} \mu^{-}$. Zeitschrift für Physik $C, 22,13-21$.

[24] The JADE Collaboration, Barte, W., et al. (1985) New Results on $e^{+} e^{-} \rightarrow \mu^{+} \mu^{-}$ from the JADE Detector at PETRA. Zeitschrift für Physik $C$, 14, 53-57.

[25] The MD-1 Collaboration, Blinov, A.E., et al. (1996) The Measurement of $R$ in $e^{+} e^{-}$ Annihilation at Center-of-Mass Energies between 7.25 and $10.34 \mathrm{GeV}$. Zeitschrift für Physik C, 70, 31-37. https://doi.org/10.1007/s002880050077

[26] Okamoto, Y., Sugita, K. and Sekine, M. (1994) Possible Removal of Preon self-Energy Divergences in CP Violation Model. IL. Nuovo Cimento, 107A, 1363 1367 https://doi.org/10.1007/BF02775775

[27] Sugita, K., Okamoto, Y. and Sekine, M. (1994) CP Violation in Preon Model. IL Nuovo Cimento, 107A, 1793-1795. https://doi.org/10.1007/BF02780713

[28] Sugita, K., Okamoto, Y. and Sekine, M. (1994) CP Violation in $\beta$ Decay. IL Nuovo Cimento, 107A, 2875-2878. https://doi.org/10.1007/BF02730965

[29] Okamoto, Y., Sugita, K. and Sekine, M. (1995) CP Violation in $\beta$ decay- II . IL Nuo- 
vo Cimento, 108A, 1153-1156. https://doi.org/10.1007/BF02790322

[30] Sekine, M., Sugita, K. and Okamoto, Y. (1997) Anomaly Freedom in CP Violation Preon Model. International Journal of Theoretical Physics, 36, 1409-1412. https://doi.org/10.1007/BF02435934

[31] Boesgaard, A. and Steigman, G. (1985) Big Bang Nucleosynthesis: Theories and Observations. Annual Review of Astronomy and Astrophysics, 23, 319-378. https://doi.org/10.1146/annurev.aa.23.090185.001535

[32] Sugita, K., Okamoto, Y. and Sekine, M. (1993) Four Families in Preon Model. IL Nuovo Cimento, 106A, 771-775. https://doi.org/10.1007/BF02771492

[33] Einstein, A. (1917) Kosmologische Betrachtungen Zur Allgemeinen Relativitätstheorie. Sitzungsberichte der Preußischen Akademie der Wissenschaften, 67, 142-152.

[34] Corda, C. (2009) Interferometric Detection of Gravitational Waves: The Definitive Test for General Relativity. International Journal of Modern Physics D, 18, 2275-2282. https://doi.org/10.1142/S0218271809015904

[35] Netchitailo, V. (2020) Hypersphere World-Universe Model: Basic Ideas. Journal of High Energy Physics, Gravitation and Cosmology, 6, 710-752. https://doi.org/10.4236/jhepgc.2020.64049

[36] Netchitailo, V. (2019) Dark Matter Cosmology and Astrophysics. Journal of High Energy Physics, Gravitation and Cosmology, 5, 999-1050.

https://doi.org/10.4236/jhepgc.2019.54056

[37] Musha, T and Sekine, M. (1976) Induced Emission of Gravitational Waves. Lettere al Nuovo Cimento, 16, 14-16. https://doi.org/10.1007/BF02719663

[38] Shandarin, S. F. and Zeldovich, Y.B. (1989) The Large-Scale Structure of the Universe: Turbulence, Intermittency, Structures in a Self-Gravitating Medium. Reviews of Modern Physics, 61,185-220. https://doi.org/10.1103/RevModPhys.61.185 